\title{
Potential Influential Factors of In-Hospital Myocardial Reinfarction in ST-Segment Elevation Myocardial Infarction (STEMI) Patients: Finding from the Improving Care for Cardiovascular Disease in China- (CCC-) Acute Coronary Syndrome (ACS) Project
}

\author{
Xiaojie Cai $(\mathbb{D}$, Juan Zhou, Wenyuan Li, Lele Cheng, Zuyi Yuan $\mathbb{D}$, and Yihui Xiao $\mathbb{D}$ \\ Department of Cardiovascular Medicine, First Affiliated Hospital of Xi'an Jiaotong University, Xi'an, China \\ Correspondence should be addressed to Zuyi Yuan; zuyiyuan@xjtu.edu.cn and Yihui Xiao; sbw_514@163.com
}

Received 6 March 2021; Revised 15 August 2021; Accepted 26 August 2021; Published 6 October 2021

Academic Editor: Ying Han

Copyright ( 2021 Xiaojie Cai et al. This is an open access article distributed under the Creative Commons Attribution License, which permits unrestricted use, distribution, and reproduction in any medium, provided the original work is properly cited.

In this study, 39915 inpatients with a discharge diagnosis of STEMI from the CCC-ACS project phase I and II were included. The prevalence of the medical history, clinical complications on admission and treatment during hospitalization in the STEMI inpatients with and without in-hospital reinfarction was presented. The factors that were differentially distributed and of critical clinical significance (e.g., age, sex, heart rate, smoking, MI history, HF history, COPD history, stroke, hypertension, diabetes, PCI treatment, administration of DAPT, and statins) were entered into standard Cox regression model and competing risk model for potential influential factors of in-hospital reinfarction. Patients with a higher heart rate (OR 1.018; 95\% CI 1.003 to 1.033 ) were more susceptible to in-hospital reinfarction. Myocardial infarction history (OR 2.840; $95 \%$ CI 1.160 to 6.955 ) was a risk factor of in-hospital reinfarction independent of hypertension, diabetes, and dyslipidaemia.

\section{Introduction}

Cardiovascular disease (CVD) remains a public health concern, causing death in $40 \%$ of the Chinese population [1]. Acute coronary syndrome (ACS), especially ST-segment elevation myocardial infarction (STEMI), is the most devastating clinical manifestation of CVD [2-5]. The prognosis of STEMI varies among individuals [6-9]. Reinfarction, major adverse cardiovascular event (MACE), sharply increases mortality among STEMI patients $[10,11]$. In previous studies, Steen et al. tried to identify the risk factors of myocardial reinfarction after anaesthesia and surgery by analysing 587 patients during 1974 and 1975 at their institution who had a myocardial infarction history and suggested that postoperative intensive care unit admission did not significantly affect the reinfarction rate, nor did diabetes, angina, patient age, sex, or site of the previous myocardial infarction [12]. A randomized controlled trial with a factorial design was performed to examine the effects of dietary intervention in the secondary prevention of myocardial infarction (MI) and stated that the 2-year incidence of reinfarction plus death from ischaemic heart disease was not significantly affected by any of the dietary regimens [13]. Recently, some studies have defined reinfarction as a main outcome measure and tried to explore the relation between treatment and reinfarction [14-16]. However, few studies have focused on this high-risk population and performed a systematic evaluation.

People in China started paying attention to medical care for CVD in the 1970s [17]. Over the past decades, clinicians and epidemiologists have been trying to improve and standardize care for cardiovascular disease $[18,19]$. In 2014, to estimate the prevalence and improve care for cardiovascular disease in China, the American Heart Association (AHA) and Chinese Society of Cardiology (CSC) launched a nationwide registry and quality improvement study in 


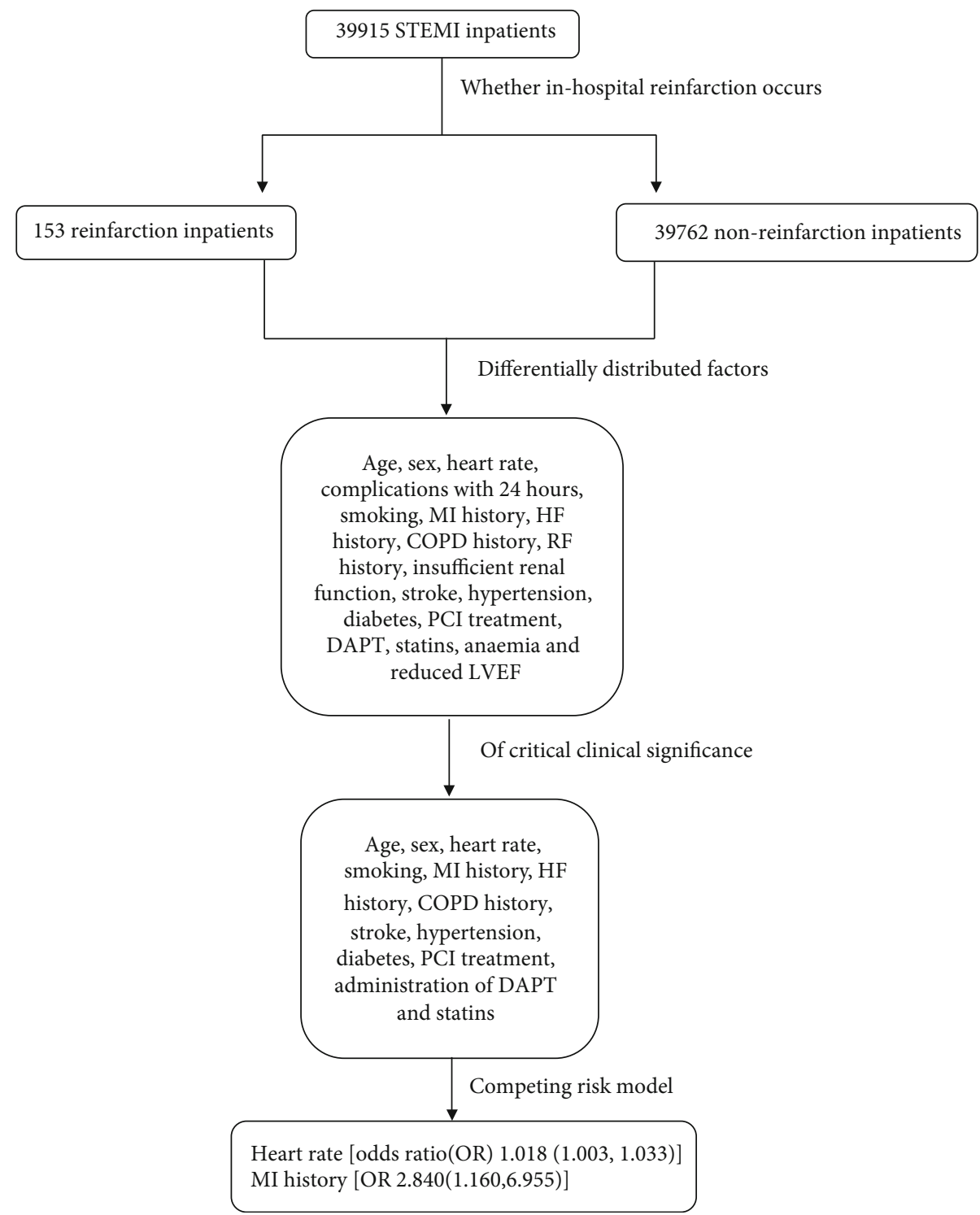

FIgURE 1: Workflow.

China-Care for Cardiovascular Disease in China (CCC) project. A total of 150 tertiary hospitals from 30 provinces were included in this project, which consists of the acute coronary syndrome (CCC-ACS) project and atrial fibrillation (CCC-AF) project. From November 2014 to June 2017, 63641 ACS inpatients were registered in the CCC-ACS project. Details of the design and methodology of the CCC project have been published previously [20].

Data from 39915 STEMI patients participating in the CCC-ACS project were used in this study. We first provide the prevalence of medical history and clinical complications on admission and treatment during hospitalization of the STEMI inpatients with and without in-hospital reinfarction. The factors that were differentially distributed and of critical clinical significance were entered into standard Cox regression and competing risk models to identify potential influential factors of in-hospital reinfarction (Figure 1: workflow). The study benefited from the large population size and appreciable geographical distribution within China. We think that the data we showed in this study comprehensively fill a gap in the knowledge about the in-hospital reinfarction population.

\section{Study Design and Methods}

Institutional review board approval was granted for the aggregate data set for research and quality improvement by the Ethics Committee of Beijing An Zhen Hospital, Capital Medical University. Participating sites were granted a waiver of patient consent under the common rule. Thirty-nine sites received institutional review board approval from their own ethics committees, with the other 111 sites accepting central ethics approval. The study is registered at http://www .clinicaltrials.gov/ (NCT02306616).

2.1. Study Population. The CCC-ACS project, whose aim is to improve and evaluate the quality of care for ACS inpatients, is a nationwide quality improvement registry 
programme launched in 2014. Hospitals were recruited to represent the diversity of ACS care in tertiary hospitals in different geographic-economic regions in China. In each geographical region of the seven regions in China: Northern, Northeast, Eastern, Central, Southern, Southwest, and Eastern China, provinces are grouped into quartiles according to gross domestic product per capita, namely, low, medium-low, medium-high, and high levels. In each geographic-economic region, $10 \%$ of the tertiary hospitals were recruited for our study, with 150 hospitals selected in phases I and II. The rationale and design of the study have been published previously. Briefly, from the 150 recruited tertiary hospitals in phases I and II, the first 20 to 30 inpatients at each hospital were consecutively recruited for the CCC-ACS project. In this study, we included inpatients with a discharge diagnosis of STEMI. STEMI was defined strictly in compliance with the guidelines issued by the CSC for diagnosis and management [21-23]. The diagnostic criteria for STEMI were based on chest pain or discomfort, electrocardiogram (ECG), and measurements of myocardial injury biomarkers. From November 2014 to June 2017, 39915 STEMI inpatients were registered in the CCC-ACS project phases I and II.

2.2. Data Collection. In this study, a standard web-based data collection platform (Oracle Clinical Remote Data Capture, Oracle) was applied for trained data abstractors at the participating hospitals to report the required data from original medical records. Each month, eligible patients were consecutively reported to the CCC-ACS database before the middle of the following month. The data have been made available for onsite audits by third parties for quality control. According to the quality audit reports, the data in this study were properly reported with an accuracy rate greater than 95\%. Data elements collected in this study included patients' demographics, medical history, symptoms on arrival, in-hospital treatments and procedures, discharge medications, and secondary prevention strategies.

2.3. Definition of STEMI. STEMI is defined as persistent chest discomfort or other symptoms suggestive of ischaemia and ST-segment elevation in at least two continuous leads $[4,24]$.

2.4. Definition of In-Hospital Reinfarction. We defined inhospital myocardial infarction as a second acute myocardial infarction during hospitalization in STEMI inpatients, including reinfarction and recurrent infarction. Time to event was defined as the time difference between (1) the date of reinfarction and the date of admission for reinfarction patients, (2) the date of death and the date of admission for patients who died during this hospitalization, and (3) the date of hospital discharge and the date of admission for those who had neither of them [25].

2.5. Study Variables. Admission complications were defined as the symptoms complicating this attack within 24 hours, including cardiac shock (CS), heart failure (HF), and cardiac arrest (CA). Current smoking was defined as smoking in the last year. Diabetes was defined as a medical history of diabetes or use of glucose-lowering therapy before hospitalization or having a fasting blood glucose level $\geq 7.0 \mathrm{mmol} / \mathrm{L}$ $(126 \mathrm{mg} / \mathrm{dL})$ or glycated haemoglobin A1c (HbA1c) concentration $\geq 6.5 \%$. Hypertension was defined as having a history of hypertension, use of antihypertensive therapy, or systolic blood pressure $(\mathrm{SBP}) \geq 140 \mathrm{mmHg}$ or diastolic blood pressure (DBP) $\geq 90 \mathrm{mmHg}$ at admission. Dyslipidaemia was defined as a history of dyslipidaemia or serum lowdensity lipoprotein cholesterol (LDL-C) $\geq 1.8 \mathrm{mmol} / \mathrm{L}$ $(70 \mathrm{mg} / \mathrm{dL})$ or serum high-density lipoprotein cholesterol ( HDL-C) $<1.0 \mathrm{mmol} / \mathrm{L}(40 \mathrm{mg} / \mathrm{dL})$ or serum triglyceride (TG )$\geq 2.3 \mathrm{mmol} / \mathrm{L}(200 \mathrm{mg} / \mathrm{dL})$. Insufficient renal function was defined as an estimated glomerular filtration rate $<60 \mathrm{~mL}$. $\min ^{-1} \cdot 1.73 \mathrm{~m}^{-2}$. Anaemia was defined as having haemoglobin $<120 \mathrm{~g} / \mathrm{L}$ for males and $<110 \mathrm{~g} / \mathrm{L}$ for females. Reduced left ventricular ejection fraction (LVEF) was defined as having an $\mathrm{LVEF}<50 \%$. A history of coronary heart disease (CHD) was defined as a history of MI or percutaneous coronary intervention (PCI) or coronary artery bypass grafting (CABG) before the current hospitalization. The transfer status indicated whether the patient was transferred from another hospital. Cerebrovascular disease, heart failure (HF), peripheral artery disease (PAD), atrial fibrillation (AF), and renal failure (RF) were defined according to the notes on original medical records. Acute management included fibrinolysis, PCI, and medicine given within 24 hours on admission via oral/intravenous administration, including dual antiplatelet therapy (DAPT), anticoagulants, statins, $\beta$-blockers, and angiotensin-converting enzyme inhibitors (ACEIs)/angiotensin-receptor blockers (ARBs) [26].

2.6. Statistical Analysis. Continuous variables with a normal distribution were shown as the mean (standard deviation $(\mathrm{SD})$ ), and differences between groups were compared using $t$-tests; continuous variables with a skewed distribution were shown as the median (interquartile range (IQR)) and were compared using the Mann-Whitney $U$ test; categorical variables were presented as the percentage (number) and were compared using the chi-square test. Baseline characteristics were described and included vital signs, symptoms at arrival, medical history, and treatment during hospitalization, i.e., age (continuous), sex (male/female), systolic blood pressure (SBP; continuous), diastolic blood pressure (DBP; continuous), heart rate (HR; continuous), cardiac complications on admission (yes/no), Killip class on admission (class I/II-III/IV), current smoking (yes/no), history of MI (yes/no), HF (yes/no), chronic obstructive pulmonary disease (COPD; yes/no), cerebrovascular disease (stroke; yes/no), peripheral artery disease (PAD; yes/no), renal failure (RF; yes/no), administration of DAPT (yes/no), anticoagulant (yes/no), statins (yes/no), $\beta$-blockers (yes/no), ACEIs/ARBs (yes/no) during hospitalization, PCI treatment (yes/no), and whether patients were transferred from another hospital before the current hospitalization (yes/no).

We used a standard Cox survival regression and a competing risk regression that considered in-hospital death as a competing risk event to address potential risk factors (e.g., 
TABLE 1: Clinical characteristics of STEMI patients on admission.

\begin{tabular}{|c|c|c|c|c|}
\hline Variables & Total $(n=39915)$ & Reinfarction $(n=153)$ & Non-reinfarction $(n=39762)$ & $p$ value \\
\hline Female (\%) $(n)$ & $21.7(8678)$ & $34(52)$ & $21.7(8626)$ & $<0.001$ \\
\hline Age (y), mean (SD) & $61.65(12.6)$ & $67.60(11.1)$ & $61.63(12.6)$ & $<0.001$ \\
\hline BMI, median (quartile) & $24.34(3.63)$ & $23.80(4.01)$ & $24.34(3.62)$ & 0.208 \\
\hline SBP $(\mathrm{mmHg})$, mean $(\mathrm{SD})$ & $127.11(23.4)$ & $129.58(26.1)$ & $127.09(23.4)$ & 0.242 \\
\hline DBP (mmHg), mean (SD) & $77.55(14.7)$ & $77.52(15.4)$ & $77.55(14.7)$ & 0.981 \\
\hline HR (bpm), mean (SD) & $77.97(16.5)$ & $82.94(17.9)$ & $77.96(16.5)$ & 0.001 \\
\hline Cardiac shock (\%) (n) & $4.1(1628)$ & $11.8(18)$ & $4.0(1610)$ & $<0.001$ \\
\hline Heart failure (\%) (n) & $8.9(3511)$ & $18.0(27)$ & $8.9(3484)$ & $<0.001$ \\
\hline Cardiac arrest $(\%)(n)$ & $2.7(1046)$ & $7.9(12)$ & $2.6(1034)$ & 0.001 \\
\hline Killip class $(\%)(n)$ & & & & $<0.001$ \\
\hline I & $70.6(26853)$ & $60(90)$ & $70.6(26763)$ & - \\
\hline II-III & $24.8(9428)$ & $28.0(42)$ & $24.8(9386)$ & - \\
\hline IV & $4.6(1754)$ & $12.0(18)$ & $4.6(1736)$ & - \\
\hline Transfer (\%) (n) & $51.6(20598)$ & $43.1(66)$ & $51.6(20532)$ & 0.036 \\
\hline Mortality (\%) (n) & $2.3(905)$ & $30.7(47)$ & $2.2(858)$ & $<0.001$ \\
\hline Smoking (\%) $(n)$ & $47.3(18885)$ & $37.9(58)$ & $47.3(18827)$ & 0.002 \\
\hline MI history (\%) (n) & $5.2(2071)$ & $20.3(31)$ & $5.1(2040)$ & $<0.001$ \\
\hline PCI history $(\%)(n)$ & $4.5(1788)$ & $15.0(23)$ & $4.4(1765)$ & $<0.001$ \\
\hline CABG history $(\%)(n)$ & $0.2(90)$ & $0.7(1)$ & $0.2(89)$ & 0.791 \\
\hline CHD family history (\%) (n) & $2.5(1008)$ & $1.3(2)$ & $2.5(1006)$ & 0.481 \\
\hline Atrial fibrillation (\%) $(n)$ & $1.5(582)$ & $2.0(3)$ & $1.5(579)$ & 0.856 \\
\hline Heart failure history $(\%)(n)$ & $0.9(378)$ & $5.9(9)$ & $0.9(369)$ & 0.001 \\
\hline COPD history $(\%)(n)$ & $1.3(523)$ & $4.6(7)$ & $1.3(516)$ & 0.001 \\
\hline Renal failure (\%) (n) & $1.1(425)$ & $3.9(6)$ & $1.1(419)$ & 0.001 \\
\hline Insufficient renal function (\%) $(n)$ & $22.3(5818)$ & $37.0(37)$ & $22.3(5781)$ & $<0.001$ \\
\hline Valvular disease history (\%) $(n)$ & $0.1(41)$ & $0.7(1)$ & $0.1(40)$ & 0.386 \\
\hline Peripheral artery disease (\%) $(n)$ & $0.6(247)$ & $0.7(1)$ & $0.6(246)$ & 1 \\
\hline Stroke $(\%)(n)$ & $8.6(3447)$ & $14.4(22)$ & $8.6(3425)$ & 0.011 \\
\hline Hypertension (\%) (n) & $64.2(25456)$ & $75.2(115)$ & $64.1(25341)$ & 0.004 \\
\hline Diabetes $(\%)(n)$ & $47.0(17436)$ & $56.4(79)$ & $47.0(17357)$ & 0.026 \\
\hline Elevated fasting glucose (\%) $(n)$ & $35.2(12817)$ & $42.4(56)$ & $35.2(12761)$ & 0.082 \\
\hline Elevated HbA1c (\%) $(n)$ & $37.2(13868)$ & $43.1(59)$ & $37.2(13809)$ & 0.157 \\
\hline Dyslipidaemia (\%) $(n)$ & $95.8(35292)$ & $95.0(134)$ & $95.8(35158)$ & 0.655 \\
\hline Elevated LDL (\%) $(n)$ & $86.8(31222)$ & $86.6(116)$ & $86.8(31106)$ & 0.946 \\
\hline Lowered HDL (\%) (n) & $46.2(16668)$ & $36.3(49)$ & $46.2(16619)$ & 0.021 \\
\hline Elevated TG (\%) $(n)$ & $18.6(6765)$ & $12.6(17)$ & $18.7(6748)$ & 0.071 \\
\hline Anaemia $(\%)(n)$ & $19.8(7262)$ & $32.6(46)$ & $19.8(7216)$ & $<0.001$ \\
\hline Reduced LVEF (\%) $(n)$ & $31.2(9637)$ & $50.9(57)$ & $31.3(9580)$ & $<0.001$ \\
\hline
\end{tabular}

Abbreviations: $\mathrm{OR}=$ odds ratio; $\mathrm{CI}=$ confidence interval; $\mathrm{BMI}=$ body mass index; $\mathrm{SBP}=$ systolic blood pressure; $\mathrm{DBP}=$ diastolic blood pressure; $\mathrm{HR}=$ heart rate; $\mathrm{MI}=$ myocardial infarction; $\mathrm{PCI}=$ percutaneous coronary intervention; $\mathrm{CABG}=$ coronary artery bypass grafting; $\mathrm{CHD}=$ coronary heart disease; $\mathrm{COPD}=$ chronic obstructive pulmonary disease; $\mathrm{LDL}=$ low-density lipoprotein; $\mathrm{HDL}=$ high-density lipoprotein; $\mathrm{TG}=$ triglyceride .

age, sex, heart rate, smoking, MI history, HF history, COPD history, stroke, hypertension, diabetes, PCI treatment, administration of DAPT, and statins) of in-hospital reinfarction (the framework can be seen in Figure 1).

Statistical analyses were performed using SPSS 22.0 (IBM, USA) and Stata 14.2 (Stata, College Station, TX, USA). Two-tailed $p$ values of less than 0.05 were considered indicative of statistical significance.

\section{Results}

3.1. Patient Characteristics. Among the 39915 STEMI inpatients registered in the CCC-ACS project, 153 experienced in-hospital myocardial reinfarction. The medical characteristics on admission are presented in Table 1. In-hospital reinfarction increases the mortality rate from $2.2 \%$ to $30.7 \%$. The reinfarction population had an average age of 
TABLE 2: Acute management within 24 hours of admission.

\begin{tabular}{|c|c|c|c|c|}
\hline Variables & Total $(n=39915)$ & Reinfarction $(n=153)$ & Nonreinfarction $(n=39762)$ & $p$ \\
\hline Fibrinolysis (\%) $(n)$ & $3.6(1350)$ & $8.7(11)$ & $3.6(1339)$ & 0.005 \\
\hline PCI $(\%)(n)$ & $83.7(30026)$ & $73.7(84)$ & $83.8(29942)$ & 0.005 \\
\hline Reperfusion (\%) (n) & $85.2(34860)$ & $80.0(88)$ & $85.2(29616)$ & 0.123 \\
\hline DAPT $(\%)(n)$ & $95.0(37922)$ & $90.2(138)$ & $95.0(37784)$ & 0.006 \\
\hline Aspirin (\%) (n) & $96.0(38295)$ & $92.2(141)$ & $96.0(38154)$ & 0.017 \\
\hline P2Y12 inhibitor (\%) $(n)$ & $96.7(38591)$ & $93.5(143)$ & $96.7(38448)$ & 0.024 \\
\hline Clopidogrel (\%) $(n)$ & $76.0(30337)$ & $75.8(116)$ & $76.0(30211)$ & 0.989 \\
\hline Ticagrelor (\%) (n) & $26.2(10436)$ & $22.9(35)$ & $26.2(10401)$ & 0.354 \\
\hline$\beta$-Blocker (\%) (n) & $53.3(21246)$ & $53.6(82)$ & $53.2(21164)$ & 0.932 \\
\hline ACEI/ARB (\%) (n) & $46.7(18636)$ & $42.5(65)$ & $46.7(18571)$ & 0.293 \\
\hline Statin $(\%)(n)$ & $93.9(37482)$ & $89.5(137)$ & $94.0(37345)$ & 0.022 \\
\hline GP IIb/IIa receptor blockers (\%) (n) & $38.9(15503)$ & $35.9(55)$ & $38.9(15448)$ & 0.461 \\
\hline Anticoagulants $(\%)(n)$ & $79.3(31626)$ & $78.4(120)$ & $79.3(31506)$ & 0.799 \\
\hline
\end{tabular}

$67.60 \pm 11.1$ years old, and $34 \%$ were women. In the STEMI patients who did not experience reinfarction in the hospital, the average age was $61.63 \pm 12.6$ years old, and the proportion of women was $21.7 \%$. A higher heart rate was observed in reinfarction patients $(82.94 \pm 17.9$ vs. $77.96 \pm 16.5 \mathrm{bpm}$, $p<0.001)$. The prevalence of severe clinical complications, including HF $(18.0 \%$ vs. $8.9 \%, p<0.001)$, CS $(11.8 \%$ vs. $4.1 \%, p<0.001)$, and CA $(7.9 \%$ vs. $2.6 \%, p<0.001)$, was higher in the reinfarction patients. With regard to cardiac function on admission, $60 \%$ of the patients with reinfarction were in Killip class I, $28.0 \%$ were in II-III, and $12 \%$ were in IV; among patients without in-hospital reinfarction, 70.6\% were in I, $24.8 \%$ were in II-III, and $4.6 \%$ were in IV.

In terms of medical history, reinfarction patients had a higher prevalence of MI history $(20.3 \%$ vs. $5.2 \%, p<0.001$ ), HF history (5.9\% vs. $0.9 \%, p=0.001)$, COPD history ( $4.6 \%$ vs. $1.3 \%, p=0.001)$, RF history $(3.9 \%$ vs. $1.1 \%, p=$ $0.001)$, stroke $(14.4 \%$ vs. $8.6 \%, p=0.012)$, hypertension ( $75.2 \%$ vs. $64.1 \%, p=0.002)$, diabetes $(56.4 \%$ vs. $47.0 \%, p$ $=0.028)$, anaemia $(32.6 \%$ vs. $19.8 \%, p<0.001)$, and reduced LVEF $(50.9 \%$ vs. $31.2 \%, p<0.001)$. Moreover, the proportions of CHD family history $(1.3 \%$ vs. $2.5, p=0.481)$, atrial fibrillation history $(2.0 \%$ vs. $1.5 \%, p=0.856)$, valvular disease history $(0.7 \%$ vs. $0.1 \%, p=0.386)$, peripheral artery disease history $(0.7 \%$ vs. $0.6 \%, p=1.000)$, and dyslipidaemia (95.0\% vs. $95.8 \%, p=0.655)$ were found to be identical between patients with and without reinfarction.

3.2. Acute Management. The proportion of eligible patients with in-hospital reinfarction who received DAPT and statins at arrival and during hospitalization was lower than that who did not experience reinfarction $(90.2 \%$ vs. $95.0 \%$ $(p<0.006)$ and $89.5 \%$ vs. $93.9 \%(p=0.022)$, respectively). Regarding revascularization, among patients with reinfarction, $80.0 \%$ received acute reperfusion therapy, $73.7 \%$ underwent primary PCI, and $8.7 \%$ received fibrinolytic therapy; the proportion of those without reinfarction was $85.2 \%$, $83.7 \%$, and $3.6 \%$, respectively. The proportions of ACEI/ARB, $\beta$-blocker, and anticoagulant use were compara- ble between patients with and without reinfarction $(42.5 \%$ vs. $46.7 \%(p=0.293), 53.6 \%$ vs. $53.2 \%(p=0.293)$, and $78.4 \%$ vs. $79.3 \%(p=0.799)$, respectively) (Table 2$)$.

3.3. Heart Rates and Myocardial Infarction History Could Be Potential Influential Factors of In-Hospital Reinfarction. Univariate Cox regression showed that age, sex, heart rate, complications within 24 hours, smoking, MI history, HF history, COPD history, RF history, insufficient renal function, stroke, hypertension, diabetes, PCI treatment, DAPT, statins, anaemia, and reduced LVEF differed between patients with and without reinfarction. On the basis of clinical significance and a limited reinfarction population, we entered age, sex, heart rate, smoking, MI history, HF history, COPD history, stroke, hypertension, diabetes, PCI, DAPT, and statin use into Cox regression models. Competing risk analysis showed that patients with a higher heart rate (OR 1.018; $95 \%$ CI 1.003 to 1.033 ) were more susceptible to inhospital reinfarction, and myocardial infarction history (OR 2.840; 95\% CI 1.160 to 6.955) was a risk factor of inhospital reinfarction independent of hypertension, diabetes, and dyslipidaemia (Table 3).

\section{Discussion}

In this study, 39915 STEMI patients from 150 hospitals throughout China who were registered in the CCC-ACS project were included. Patients with in-hospital reinfarction were characterized in a comprehensive manner. We presented the demographics, clinical condition on admission, medical history, and care during hospitalization of STEMI patients with and without in-hospital reinfarction. In general, patients with reinfarction had advanced age, worse cardiac function, and a history of CHD. A competing risk model showed that a higher heart rate (OR 1.018 (1.003, 1.033)) and myocardial infarction history (OR 2.840 $(1.160,6.955))$ could be potential influential factors of inhospital reinfarction. The study benefited from the large population size and appreciable geographic distribution 
TABLE 3: Adjusted odds ratios from standard Cox analysis and competing risk analysis.

\begin{tabular}{|c|c|c|c|c|}
\hline Variables & OR $(95 \% \mathrm{CI})$ & $p$ & Adjusted OR (95\% CI) & Adjusted $p$ \\
\hline Age & $1.02(0.994,1.046)$ & 0.138 & $1.019(0.998,1.041)$ & 0.082 \\
\hline Sex & $1.565(0.785,3.12)$ & 0.203 & $1.56(0.749,3.248)$ & 0.235 \\
\hline Heart rate & $1.019(1.004,1.034)$ & 0.015 & $1.018(1.003,1.033)$ & 0.016 \\
\hline Smoking & $1.098(0.564,2.137)$ & 0.783 & $1.1(0.565,2.142)$ & 0.78 \\
\hline MI history & $2.84(1.204,6.699)$ & 0.017 & $2.84(1.16,6.955)$ & 0.022 \\
\hline HF history & $1.581(0.336,7.437)$ & 0.562 & $1.543(0.274,8.691)$ & 0.623 \\
\hline COPD history & $2.058(0.473,8.96)$ & 0.336 & $2.041(0.434,9.613)$ & 0.367 \\
\hline Stroke & $0.929(0.362,2.383)$ & 0.878 & $0.927(0.359,2.394)$ & 0.876 \\
\hline Hypertension & $1.116(0.576,2.164)$ & 0.745 & $1.114(0.558,2.224)$ & 0.76 \\
\hline Diabetes & $1.079(0.602,1.933)$ & 0.799 & $1.064(0.584,1.936)$ & 0.84 \\
\hline PCI & $0.766(0.387,1.596)$ & 0.506 & $0.806(0.391,1.661)$ & 0.56 \\
\hline DAPT & $2.654(0.349,20.197)$ & 0.346 & $2.665(0.392,18.122)$ & 0.316 \\
\hline Statins & $0.633(0.192,2.092)$ & 0.454 & $0.648(0.215,1.952)$ & 0.441 \\
\hline
\end{tabular}

within China. We think that the data we showed in this study comprehensively fill a gap in the knowledge of the in-hospital reinfarction population in China, though this was a case-control study with low power to predict influential factors.

An elevated heart rate is associated with adverse outcomes in the general population, as well as in patients with hypertension, stable CAD, and chronic $\operatorname{HF}[27,28]$. In previous studies, an increased resting heart rate was recognized as a risk factor for chronic heart failure [28]. Additionally, among AMI patients without heart failure, patients with lower heart rate or $\beta$-blocker therapy had a better prognosis [29]. According to this study, the OR of heart rate in the competing risk model was 1.018, meaning that every $10 \mathrm{bpm}$ increase in $\mathrm{HR}$ raises the risk of inhospital reinfarction by $18 \%$, which indicated that heart rate reduction therapy might be beneficial even for patients without heart failure. Physiologically, elevated heart rate increases oxygen demand whilst reducing coronary perfusion time, which impairs coronary blood flow. Deficiency of oxygen supply increases vascular oxidative stress and deteriorates endothelial function which is considered a key event in the development of atherosclerosis and implies a change from the normally predominant release of nitric oxide to that of endothelium-derived contracting factors [30-33]. Hemodynamic factors which are at least defined by the duration/length of the cardiac cycle and are characterized by pulsatility and frequency of mechanical stress may precipitate plaque disruption [34, 35]. To sum up, elevated heart rate potentially promotes both the development of atherosclerosis and the plaque instability. Moreover, a proangiogenic effect of heart rate reduction was displayed using bradycardic pacing in a rabbit model and enhanced vascular endothelial growth factor expression was shown to be critical in bradycardia-induced angiogenesis [36, 37]. We think that beta-blockers and the $\mathrm{I}(\mathrm{f})$ channel inhibitor ivabradine could be considered for secondary prevention in STEMI patients with high heart rates. Additional studies need to be performed to draw further conclusions.

Competing risk model analysis indicated that myocardial infarction history was a risk factor of in-hospital reinfarction independent of hypertension, diabetes, and dyslipidaemia. Assumptions could be made that patients with certain lifestyles or genetics were more vulnerable to myocardial infarction. The HMGA1 rs146052672 variant was shown to be associated with myocardial infarction susceptibility [38]. ATG7 DNA sequence variants (DSVs) and single-nucleotide polymorphisms (SNPs) identified in AMI patients may alter the transcriptional activity of the ATG7 gene promoter and change ATG7 levels, contributing to AMI development as a rare risk factor [39]. Similarly, DSVs and SNPs of SIRT5 were suggested to contribute to AMI development as a risk factor [40]. Likewise, people who have a history of circadian rhythm disorders, eating disorders, depression, and so forth were suggested to have a high risk of myocardial infarction [41-43]. More studies need to be carried out to address genetic and environmental determinants of myocardial infarction for better prevention.

\section{Conclusion}

From the data we obtained, we concluded that heart rate and myocardial infarction history might be potential influential factors of in-hospital reinfarction.

\section{Abbreviations}

STEMI: ST-segment elevation myocardial infarction

CCC: Improving Care for Cardiovascular Disease in China

ACS: Acute coronary syndrome

MI: $\quad$ Myocardial infarction

COPD: Chronic obstructive pulmonary disease

DAPT: Dual antiplatelet therapy

HR: Heart rate 
CVD: Cardiovascular disease

MACE: Major adverse cardiovascular event

AMI: Acute myocardial infarction

AHA: American Heart Association

CSC: Chinese Society of Cardiology

AF: Atrial fibrillation

ECG: Electrocardiogram

CS: $\quad$ Cardiac shock

HF: Heart failure

CA: $\quad$ Cardiac arrest

HbA1c: Glycated haemoglobin A1c

SBP: $\quad$ Systolic blood pressure

DBP: Diastolic blood pressure

LDL-C: Low-density lipoprotein cholesterol

HDL-C: High-density lipoprotein cholesterol

TG: $\quad$ Triglyceride

LVEF: Left ventricular ejection fraction

CHD: Coronary heart disease

PCI: Percutaneous coronary intervention

CABG: Coronary artery bypass graft

PAD: Periphery artery disease

RF: $\quad$ Renal failure

ACEIs: Angiotensin-converting enzyme inhibitors

ARBs: Angiotensin-receptor blockers

SD: $\quad$ Standard deviation

IQR: Interquartile range.

\section{Data Availability}

As a collaborative initiative of the American Heart Association (AHA) and the Chinese Society of Cardiology (CSC), the CCC-ACS project collected in-hospital data of ACS patients from 150 hospitals all over China. Rationale and design of it have been published previously. (1) Briefly, 150 tertiary hospitals from different geographic and economic regions of China registered in this project. In each hospital, the first 20 to 30 inpatients are consecutively recruited to the study. STEMI was defined strictly complied with the guidelines issued by CSC for diagnosis and management. (2-4) The diagnostic criteria for STEMI were based on chest pain or discomfort, ECG, and measurements of myocardial injury biomarkers. From November 2014 to June 2017, 39915 STEMI inpatients were registered. (1) Hao Y, Liu J, Liu J, et al. Rationale and design of the Improving Care for Cardiovascular Disease in China (CCC) project A national effort to prompt quality enhancement for acute coronary syndrome. Am Heart J. 2016; 179: 107-15. (2) Chinese Society of Cardiology. Guideline for diagnosis and treatment of patients with ST-elevation myocardial infarction 2010. Chin J Cardiol. 2010; 38: 675-690. (3) Chinese Society of Cardiology. 2019 Chinese Society of Cardiology (CSC) guidelines for the diagnosis and management of patients with STsegment elevation myocardial infarction. Chin J Cardiol. 2019; 47 (10): 766-783. (4) Chinese Society of Cardiology. Guidelines for the management of acute coronary syndromes in patients presenting without persistent STsegment elevation 2012. Chin J Cardiol. 2012; 40: 353-367.

\section{Conflicts of Interest}

The authors declare that they have no competing interests.

\section{Authors' Contributions}

Xiaojie Cai and Yihui Xiao contributed equally to this article. Yihui Xiao conceived the idea. Xiaojie Cai performed data analysis. Xiaojie Cai and Yihui Xiao are responsible to write and revise the article. Juan Zhou, Wenyuan Li, and Lele Cheng participated in the interpretation of the results. Zuyi Yuan supervised the whole project.

\section{Acknowledgments}

The Improving Care for Cardiovascular Disease in China(CCC-) ACS project is a collaborative study of the American Heart Association and Chinese Society of Cardiology. The American Heart Association was funded by AstraZeneca and Pfizer for the quality improvement initiative through an independent grant for learning and change. We acknowledge the contribution of all investigators at the hospital participating in the CCC project.

\section{References}

[1] D. Zhao, J. Liu, M. Wang, X. Zhang, and M. Zhou, "Epidemiology of cardiovascular disease in China: current features and implications," Nature Reviews Cardiology, vol. 16, no. 4, pp. 203-212, 2019.

[2] J. N. Van Der Sijde, A. Karanasos, M. Villiger, B. E. Bouma, and E. Regar, "First-in-man assessment of plaque rupture by polarization-sensitive optical frequency domain imagingin vivo," European Heart Journal, vol. 37, no. 24, p. 1932, 2016.

[3] D. M. Leistner, N. Kränkel, D. Meteva et al., "Differential immunological signature at the culprit site distinguishes acute coronary syndrome with intact from acute coronary syndrome with ruptured fibrous cap: results from the prospective translational OPTICO-ACS study," European Heart Journal, vol. 41, no. 37, pp. 3549-3560, 2020.

[4] B. Ibanez, S. James, S. Agewall et al., "2017 ESC guidelines for the management of acute myocardial infarction in patients presenting with ST-segment elevation," European Heart Journal, vol. 39, no. 2, pp. 119-177, 2018.

[5] J. L. Anderson, "2013 ACCF/AHA guideline for the management of ST-elevation myocardial infarction: a report of the American College of Cardiology Foundation/American Heart Association Task Force on practice guidelines," Circulation, vol. 127, no. 4, pp. 362-425, 2013.

[6] N. R. Smilowitz, A. M. Mahajan, M. T. Roe et al., "Mortality of myocardial infarction by sex, age, and obstructive coronary artery disease status in the ACTION registry-GWTG (acute coronary treatment and intervention outcomes network registry-get with the guidelines)," Circulation. Cardiovascular Quality and Outcomes, vol. 10, no. 12, pp. 1-8, 2017.

[7] E. Cenko, J. Yoon, S. Kedev et al., "Sex differences in outcomes after STEMI," JAMA Internal Medicine, vol. 178, no. 5, pp. 632-639, 2018.

[8] S. Karayiannides, A. Norhammar, O. Frøbert, S. K. James, B. Lagerqvist, and P. Lundman, "Prognosis in patients with 
diabetes mellitus and STEMI undergoing primary PCI," Journal of the American College of Cardiology, vol. 72, no. 12, pp. 1427-1428, 2018.

[9] E. Kedhi, E. Fabris, M. van der Ent et al., "Six months versus 12 months dual antiplatelet therapy after drug-eluting stent implantation in ST-elevation myocardial infarction (DAPTSTEMI): randomised, multicentre, non-inferiority trial," $B M J$, vol. 363, p. k3793, 2018.

[10] B. Vogel, B. E. Claessen, S. V. Arnold et al., "ST-segment elevation myocardial infarction," Nature Reviews Disease Primers, vol. 5, no. 1, p. 41572, 2019.

[11] S. S. Jolly, S. James, V. Džavík et al., "Thrombus aspiration in ST-segment-elevation myocardial Infarction," Circulation, vol. 135, no. 2, pp. 143-152, 2017.

[12] P. A. Steen, J. H. Tinker, and S. Tarhan, "Myocardial reinfarction after anesthesia and surgery," JAMA: The Journal of the American Medical Association, vol. 239, no. 24, pp. 25662570, 1978.

[13] M. L. Burr, J. F. Gilbert, R. M. Holliday et al., "Effects of changes in fat, fish, and fibre intakes on death and myocardial reinfarction: diet and reinfarction trial (Dart)," Lancet, vol. 334, no. 8666, pp. 757-761, 1989.

[14] E. S. Stauffer, "Lumbar spine surgery," The Journal of Bone \& Joint Surgery, vol. 70, no. 6, p. 958, 1988.

[15] S. Yusuf, "Effects of fondaparinux on mortality and reinfarction in patients with acute ST-segment elevation myocardial infarction: the OASIS- 6 randomized trial," Journal of the American Medical Association, vol. 295, no. 13, pp. 15191530, 2006.

[16] Z. Chen and J. Xie, "Early intravenous then oral metoprolol in 45852 patients with acute myocardial infarction: randomised placebo-controlled trial," Lancet, vol. 366, no. 9497, pp. 1622-1632, 2005.

[17] Y. Wu, "Yingkai Wu-obituary," Obituary, vol. 363, 2004.

[18] Z. Wu, C. Yao, D. Zhao et al., "Sino-MONICA project: a collaborative study on trends and determinants in cardiovascular diseases in China, Part i: morbidity and mortality monitoring," Circulation, vol. 103, no. 3, pp. 462-468, 2001.

[19] H. Li and J. Ge, "Cardiovascular diseases in China: current status and future perspectives," IJC Heart \& Vasculature, vol. 6, pp. 25-31, 2015.

[20] Y. Hao, J. Liu, J. Liu et al., vol. 179, pp. 107-115, 2016.

[21] China Society of Cardiology of Chinese Medical Association and Editorial Board of Chinese Journal of Cardiology, "Guideline for diagnosis and treatment of patients with ST-elevation myocardial infarction," Zhonghua Xin Xue Guan Bing Za Zhi, vol. 38, no. 8, pp. 675-690, 2010.

[22] China Society of Cardiology of Chinese Medical Association and Editorial Board of Chinese Journal of Cardiology, "Guideline of non-ST segment elevation acute coronary syndrome," Zhonghua Xin Xue Guan Bing Za Zhi, vol. 40, no. 5, pp. 353-367, 2012.

[23] China Society of Cardiology of Chinese Medical Association and Editorial Board of Chinese Journal of Cardiology, "2019 Chinese Society of Cardiology (CSC) guidelines for the diagnosis and management of patients with ST-segment elevation myocardial infarction," Zhonghua Xin Xue Guan Bing Za Zhi, vol. 47, no. 10, pp. 766-783, 2019.

[24] J. Vojáček, P. Janský, and T. Janota, “Third universal definition of myocardial infarction," Cor et Vasa, vol. 55, no. 3, pp. e228e235, 2013.
[25] S. Gourgou-Bourgade, D. Cameron, P. Poortmans et al., "Guidelines for time-to-event end point definitions in breast cancer trials: results of the DATECAN initiative (Definition for the Assessment of Time-to-event Endpoints in CANcer trials) ${ }^{\dagger}, "$ Annals of Oncology, vol. 26, no. 5, pp. 873-879, 2015.

[26] M. Zhou, J. Liu, Y. Hao et al., "Prevalence and in-hospital outcomes of diabetes among patients with acute coronary syndrome in China: findings from the Improving Care for Cardiovascular Disease in China-Acute Coronary Syndrome Project," Cardiovascular Diabetology, vol. 17, no. 1, p. 147, 2018.

[27] D. Dobre, J. Kjekshus, P. Rossignol et al., "Heart rate, pulse pressure and mortality in patients with myocardial infarction complicated by heart failure," International Journal of Cardiology, vol. 271, pp. 181-185, 2018.

[28] M. Böhm, K. Swedberg, M. Komajda et al., "Heart rate as a risk factor in chronic heart failure (SHIFT): the association between heart rate and outcomes in a randomised placebocontrolled trial," Lancet, vol. 376, no. 9744, pp. 886-894, 2010.

[29] J. J. Park, S. H. Kim, S. H. Kang et al., "Differential effect of $\beta$ blockers according to heart rate in acute myocardial infarction without heart failure or left ventricular systolic dysfunction: a cohort study," Mayo Clinic Proceedings, vol. 94, no. 12, pp. 2476-2487, 2019.

[30] F. Custodis, M. Baumhäkel, N. Schlimmer et al., "Heart rate reduction by ivabradine reduces oxidative stress, improves endothelial function, and prevents atherosclerosis in apolipoprotein E-deficient mice," Circulation, vol. 117, no. 18, pp. 2377-2387, 2008.

[31] S. Kröller-Schön, E. Schulz, P. Wenzel et al., "Differential effects of heart rate reduction with ivabradine in two models of endothelial dysfunction and oxidative stress," Basic Research in Cardiology, vol. 106, no. 6, pp. 1147-1158, 2011.

[32] D. G. Harrison, "Cellular and molecular mechanisms of endothelial cell dysfunction," The Journal of Clinical Investigation, vol. 100, no. 9, pp. 2153-2157, 1997.

[33] M. Félétou and P. M. Vanhoutte, "Endothelial dysfunction: a multifaceted disorder (The Wiggers Award Lecture)," American Journal of Physiology-Heart and Circulatory Physiology, vol. 291, no. 3, pp. H985-H1002, 2006.

[34] R. T. Lee, F. J. Schoen, H. M. Loree, M. W. Lark, and P. Libby, "Circumferential stress and matrix metalloproteinase 1 in human coronary Atherosclerosis," Arteriosclerosis, Thrombosis, and Vascular Biology, vol. 16, no. 8, pp. 1070-1073, 1996.

[35] D. G. Katritsis, J. Pantos, and E. Efstathopoulos, "Hemodynamic factors and atheromatic plaque rupture in the coronary arteries: from vulnerable plaque to vulnerable coronary segment," Coronary Artery Disease, vol. 18, no. 3, pp. 229-237, 2007.

[36] A. J. Wright and O. Hudlicka, "Capillary growth and changes in heart performance induced by chronic bradycardial pacing in the rabbit," Circulation Research, vol. 49, no. 2, pp. 469478, 1981.

[37] W. Zheng, M. D. Brown, T. A. Brock, R. J. Bjercke, and R. J. Tomanek, "Bradycardia-induced coronary angiogenesis is dependent on vascular endothelial growth factor," Circulation Research, vol. 85, no. 2, pp. 192-198, 1999.

[38] S. de Rosa, E. Chiefari, N. Salerno et al., "HMGA1 is a novel candidate gene for myocardial infarction susceptibility," International Journal of Cardiology, vol. 227, pp. 331-334, 2017. 
[39] P. Zhang, J. Zhang, Y. Zhang, S. Wang, S. Pang, and B. Yan, "Functional variants of theATG7gene promoter in acute myocardial infarction," Molecular Genetics \& Genomic Medicine, vol. 6, no. 6, pp. 1209-1219, 2018.

[40] L. Chen, H. Wang, F. Gao et al., "Functional genetic variants in the SIRT5 gene promoter in acute myocardial infarction," Gene, vol. 675, pp. 233-239, 2018.

[41] S. S. Thosar, M. P. Butler, and S. A. Shea, "Role of the circadian system in cardiovascular disease," The Journal of Clinical Investigation, vol. 128, no. 6, pp. 2157-2167, 2018.

[42] S. Giovinazzo, S. G. Sukkar, G. M. Rosa et al., "Anorexia nervosa and heart disease: a systematic review," Eating and Weight Disorders-Studies on Anorexia, Bulimia and Obesity, vol. 24, no. 2, pp. 199-207, 2019.

[43] J. P. Headrick, J. N. Peart, B. P. Budiono, D. H. K. Shum, D. L. Neumann, and N. J. C. Stapelberg, "The heartbreak of depression: 'Psycho-cardiac' coupling in myocardial infarction," Journal of Molecular and Cellular Cardiology, vol. 106, pp. 14-28, 2017. 